Editorials

16 Morbide Adipositas

Weiner, R.A. (Frankfurt/M.); Schmidt, W.E. (Bochum)

275 Pankreaskarzinom

Klar, E. (Rostock); Seifert, H. (Oldenburg)

3154 Gallengangskarzinome

Mössner, J.; Jonas, S. (Leipzig)

4224 Neuroendokrine Tumoren des Gastrointestinaltrakts Wiedenmann, B. (Berlin); Frilling, A. (London)

Hauptthemen

\section{Morbide Adipositas}

Herausgeber: R.A. Weiner, Frankfurt/M.; W.E. Schmidt, Bochum (Hrsg.)

18 Die chirurgische Behandlung des metabolischen

\section{Syndroms}

Weiner, R.A. (Frankfurt/M.)

114 Pathophysiologie der Adipositas - neue Betrachtungsweise Blüher, M. (Leipzig)

121 Positiv-Negativ-Bilanz in der bariatrischen Chirurgie Ludwig, K.; Schneider-Koriath, S.; Bernhardt, J. (Rostock); Hüttli, T.P. (München)

126 Adipositas - welche medikamentösen Behandlungsoptionen?

Fölsch, U.R. (Kiel)

131 Endoskopische Therapie der Adipositas Fried, M.; Mertens, J.C. (Zürich)

136 Ist der laparoskopische Zugangsweg in der Adipositas-Chirurgie State of the Art? Tigges, W.; Tigges, E.P.; Pick, P. (Hamburg); Tigges, H. (Konstanz)

141 Die Beriberi, eine seltene, aber schwerwiegende nutritive Komplikation nach bariatrischen Eingriffen: Inzidenz, Symptomatologie, Therapie und Prävention Stroh, C. (Gera); Meyer, F.; Lippert, H. (Magdeburg); Manger, T. (Gera)

147 Unterschiede in der Instrumenten- und Ausstattungswahl zwischen Adipositas-Chirurgie und allgemeiner minimal invasiver Chirurgie Horbach, T.; Krüger, S. (Schwabach)

\section{Pankreaskarzinom}

Herausgeber: E. Klar, Rostock; H. Seifert, Oldenburg (Hrsg.)

278 Pankreaskarzinom: Kriterien und Grenzen der Resektabilität

Witzigmann, H.; Jungnickel, H.; Kißenkötter, S. (Dresden)

284 Minimal invasive Therapie von malignen gastroduodenalen Obstruktionen bei Inoperabilität: Stent oder Gastrojejunostomie?

Müller, M.A.; Caca, K. (Ludwigsburg)

291 Pankreaskarzinom: Algorithmus der palliativen Chemotherapie

Kullmann, F. (Weiden in der Oberpfalz)

297 Pankreaskarzinom - Standards der chirurgischen Therapie Hackert, T.; Büchler, M.W. (Heidelberg)

2103 Pankreaskarzinom: Ist die operative Therapie gerechtfertigt bei R2-Resektion, Lebermetastasen oder Rezidivtumor?

Deutsch, L.S.; Bockhorn, M.; Cataldegirmen, G.; Izbicki, J.R. (Hamburg)

2109 Neoadjuvante und adjuvante Therapiekonzepte beim duktalen Pankreaskarzinom - was gibt es Neues? Hamm, A.; Büchler, M.W.; Weitz, J. (Heidelberg)

2116 Pankreaskarzinom: Zukünftige therapeutische Perspektiven

Andrén-Sandberg, Å.; Ansorge, C.; Gilg, S. (Stockholm)

\section{Gallengangskarzinome}

Herausgeber: J. Mössner, S. Jonas, Leipzig

3158 Neue Aspekte der TNM-Klassifikation von intra- und extrahepatischen Cholangiokarzinomen Wittekind, C.; Oberschmid, B. (Leipzig)

3164 Aktuelle Chemotherapiekonzepte bei Gallengangskarzinomen Wiedmann, M. (Berlin/Leipzig)

3178 SpyGlass - neue Cholangioskope zur besseren Diagnostik?

Seelhoff, A.; Schumacher, B.; Neuhaus, H. (Düsseldorf)

3182 Therapie benigner Gallengangsstenosen nach Lebertransplantation Chahoud, F.; Gotthardt, D.; Sauer, P. (Heidelberg)

\section{KARGER}

Fax +497614520714

Information@Karger.de

www.karger.com (c) 2010 S. Karger GmbH, Freiburg 
3187 Gallengangskarzinom: Update neuer Stents zur Palliation

Schulz, H.-J. (Berlin)

3194 Gallengangskarzinom: Stellenwert der photodynamischen Therapie

Wolkersdörfer, G.W.; Kiesslich, T.; Neureiter, D.; Berr, F. (Salzburg)

3199 Lebertransplantation zur Therapie von intra- und extrahepatischen Gallengangskarzinomen Thelen, A.; Benckert, C.; Jonas, S. (Leipzig)

\section{Neuroendokrine Tumoren des Gastrointestinaltrakts}

Herausgeber: A. Frilling, London; B. Wiedenmann, Berlin

4226 Neuroendokrine Tumoren - Epidemiologie und Endokrinologie

Rinke, A.; Gress, T.M. (Marburg)

4234 Klassifikation und Pathologie gastroenteropankreatischer neuroendokriner Tumoren

Perren, A.; Schmitt, A. (Bern); Komminoth, P. (Zürich); Anlauf, M. (Düsseldorf); Klöppel, G. (München)

4242 Neuroendokrine Tumoren des Gastrointestinaltraktes: Klinik und Diagnostik Plöckinger, U. (Berlin)

\section{Chirurgische Therapie neuroendokriner}

Lebermetastasen - Resektion, Transplantation Frilling, A. (London); Sotiropoulos, G.C. (Essen); Kornasiewicz, O.; Tedeschi, M. (London); Li, J. (Tübingen)

4262 Neuroendokrine Tumoren: Lokal ablative Verfahren - RFA, LITT, Alkoholinjektion, Kryoablation Stippel, D.L. (Köln)

4269 Regionale interventionelle Behandlungsverfahren von Lebermetastasen neuroendokriner Tumoren: Embolisation (TAE), Chemoembolisation (TACE) und selektive interne Radiotherapie (SIRT) Vogl, T.J.; Nour-Eldin, N.E.A.; Zangos, S.; Grünwald, F.; Bojunga, J.; Trojan, J.; Lubomierski, N.; Gruber-Roth, T. (Frankfurt/M.)

4276 Neuroendokrine Tumoren - medikamentöse Therapie Pavel, M. (Berlin)

4283 Gastroenteropankreatische neuroendokrine Tumoren: Molekulargenetische Charakteristika Anlauf, M. (Düsseldorf); Schmitt, A. (Bern); Heikaus, S.; Schott, M.; Willenberg, H.S.; Raffel, A.; Krausch, M.; Cupisti, K.; Stoecklein, N. (Düsseldorf); Bauersfeld, J. (Zürich); Knoefel, W.T. (Düsseldorf); Komminoth, P. (Zürich); Perren, A. (Bern); Klöppel, G. (München)
Interdisziplinäres Gespräch

154 Morbide Adipositas

Manger, T. (Gera) (Discussion Leader)

2122 Pankreaskarzinome

Schoenberg, M.H. (Diskussionsleiter) (München)

\section{Gallengangskarzinome}

Heidecke, C.-D. (Greifswald) (Gesprächsleiter)

Originalarbeit

2131 Bewertung von Gesundheitsleistungen durch Laien am Beispiel der multimodalen Therapie des Kolonkarzinoms

Wachter, C.; Porzsolt, F.; Henne-Bruns, D.; Kornmann, M. (Ulm)

News / Ticker

$\mathbf{2} 139, \mathbf{3} 211,4289$

Tagungen und Kongresse

$162,2143,3170,4291$

4293 Autorenverzeichnis Band 26, 2010

4294 Sachwortverzeichnis Band 26, 2010 
Editorials

16 Morbid Obesity

Weiner, R.A. (Frankfurt/M.); Schmidt, W.E. (Bochum)

275 Pancreatic Cancer

Klar, E. (Rostock); Seifert, H. (Oldenburg)

3154 Cholangiocarcinomas

Mössner, J.; Jonas, S. (Leipzig)

4224 Neuroendocrine Tumors of the Gastrointestinal Tract

Wiedenmann, B. (Berlin); Frilling, A. (London)

Main Topic

\section{Morbid Obesity}

Editors: R.A. Weiner, Frankfurt/M.; W.E. Schmidt, Bochum

18 Surgical Treatment of the Metabolic Syndrome Weiner, R.A. (Frankfurt/M.)

114 Pathogenesis of Obesity - New Perspectives Blüher, M. (Leipzig)

121 Risk-Benefit Analysis of Bariatric Surgery Ludwig, K.; Schneider-Koriath, S.; Bernhardt, J. (Rostock); Hüttli, T.P. (München)

126 Obesity - Which Medical Treatment Options? Fölsch, U.R. (Kiel)

131 Endoscopic Therapy in Obesity Fried, M.; Mertens, J.C. (Zürich)

136 Is Laparoscopic Access State of the Art in Bariatric Surgery?

Tigges, W.; Tigges, E.P.; Pick, P. (Hamburg); Tigges, H. (Konstanz)

141 Beriberi, a Rare but Serious Nutritious Complication after Bariatric Surgical Interventions: Incidence, Symptomatology, Treatment and Prevention Stroh, C. (Gera); Meyer, F.; Lippert, H. (Magdeburg); Manger, T. (Gera)

147 Differences in the Selection of Instruments and Equipment in Bariatric Surgery and Standard Minimally Invasive Surgery Horbach, T.; Krüger, S. (Schwabach)

\section{Pankreatic Cancer}

Editors: E. Klar, Rostock; H. Seifert, Oldenburg

278 Pancreatic Cancer: Criteria and Limits of Resectability Witzigmann, H.; Jungnickel, H.; Kißenkötter, S. (Dresden)

284 Palliative Treatment of Malignant Gastric Outlet Obstruction: Stent versus Gastrojejunostomy Müller, M.A.; Caca, K. (Ludwigsburg)

291 Pancreatic Cancer: Algorithm of Palliative Chemotherapy

Kullmann, F. (Weiden in der Oberpfalz)

297 Pancreatic Cancer - Standards of Surgical Therapy Hackert, T.; Büchler, M.W. (Heidelberg)

2103 Pancreatic Cancer: Is Surgical Treatment Justified in Case of R2 Resection, Liver Metastases or Tumor Recurrence?

Deutsch, L.S.; Bockhorn, M.; Cataldegirmen, G.; Izbicki, J.R. (Hamburg)

2109 New Strategies for Neoadjuvant and Adjuvant Treatment in Ductal Adenocarcinoma of the Pancreas Hamm, A.; Büchler, M.W.; Weitz, J. (Heidelberg)

2116 Pancreatic Carcinoma: Theapeutic Future Prospects Andrén-Sandberg, Å.; Ansorge, C.; Gilg, S. (Stockholm)

Cholangiocarcinomas

Editors: Mössner, J.; Jonas, S. (Leipzig)

3158 New Aspects of TNM Classification of Tumors of the Intra- and Extrahepatic Bile Ducts Wittekind, C.; Oberschmid, B. (Leipzig)

3164 Current Concepts of Chemotherapy in Biliary Tract Cancer

Wiedmann, M. (Berlin/Leipzig)

3178 SpyGlass - New Cholangioscopes for Better Diagnostics?

Seelhoff, A.; Schumacher, B.; Neuhaus, H. (Düsseldorf)

3182 Treatment of Benign Biliary Strictures after Liver Transplantation

Chahoud, F.; Gotthardt, D.; Sauer, P. (Heidelberg)

3187 Cholangiocarcinoma: Update of New Stents for Palliation

Schulz, H.-J. (Berlin)

\section{KARGER}

Fax +497614520714

Information@Karger.de

www.karger.com (c) 2010 S. Karger GmbH, Freiburg 
3194 Photodynamic Therapy in Proximal Bile Duct Cancer

Wolkersdörfer, G.W.; Kiesslich, T.; Neureiter, D.; Berr, F. (Salzburg)

3199 Liver Transplantation for Treatment of Intra- and Extrahepatic Cholangiocarcinomas

Thelen, A.; Benckert, C.; Jonas, S. (Leipzig)

\section{Neuroendocrine Tumors of the Gastrointestinal Tract}

Editors: A. Frilling, London; B. Wiedenmann, Berlin

Main Topic

4226 Neuroendocrine Tumors - Epidemiology and Endocrinology

Rinke, A.; Gress, T.M. (Marburg)

4234 Classification of Gastroenteropancreatic Neuroendocrine Tumors

Perren, A.; Schmitt, A. (Bern); Komminoth, P. (Zürich); Anlauf, M. (Düsseldorf); Klöppel, G. (München)

4242 Neuroendocrine Tumors of the Gastrointestinal Tract: Signs, Symptoms, and Diagnostics Plöckinger, U. (Berlin)

\section{Surgical Treatment of Neuroendocrine Liver}

Metastases - Resection, Transplantation

Frilling, A. (London); Sotiropoulos, G.C. (Essen); Kornasiewicz, O.; Tedeschi, M. (London); Li, J. (Tübingen)

\section{Neuroendocrine Tumors: Local Ablative} Procedures - RFA, LITT, PEI, Cryoablation Stippel, D.L. (Köln)

4269 Regional Interventional Treatments of Liver Metastases from Neuroendocrine Tumors: Embolization (TAE), Chemoembolization (TACE) and Selective Internal Radiation Therapy (SIRT) Vogl, T.J.; Nour-Eldin, N.E.A.; Zangos, S.; Grünwald, F.; Bojunga, J.; Trojan, J.; Lubomierski, N.; Gruber-Roth, T. (Frankfurt/M.)

4276 Neuroendocrine Tumors - Medical Treatment Pavel, M. (Berlin)

4283 Gastroenteropancreatic Neuroendocrine Tumors: Molecular Genetic Features Anlauf, M. (Düsseldorf); Schmitt, A. (Bern); Heikaus, S.; Schott, M.; Willenberg, H.S.; Raffel, A.; Krausch, M.; Cupisti, K.; Stoecklein, N. (Düsseldorf); Bauersfeld, J. (Zürich); Knoefel, W.T (Düsseldorf); Komminoth, P. (Zürich); Perren, A. (Bern); Klöppel, G. (München)
Interdisciplinary Discussion

154 Morbid Obesity

Manger, T. (Gera) (Discussion Leader)

2122 Pancreatic Carcinomas

Schoenberg, M.H. (Diskussionsleiter) (München)

\section{Cholangiocarcinomas}

Heidecke, C.-D. (Greifswald) (Discussion Leader)

Original Article

2131 Lay Assessment of Health Care Services at the Example of Colon Cancer Treatment

Wachter, C.; Porzsolt, F.; Henne-Bruns, D.; Kornmann, M. (Ulm)

News / Ticker

2 139, 3211,4289

Meetings and Conferences

$\mathbf{1} 62,2143,3170,4291$

4293 Author Index Vol. 26, 2010

4295 Subject Index Vol. 26, 2010 